Article

\title{
Solutions of Extension and Limits of Some Cantorian Paradoxes
}

\author{
Josué-Antonio Nescolarde-Selva ${ }^{1, * \mathbb{D}}$, José-Luis Usó-Doménech ${ }^{1} \mathbb{D}$, Lorena Segura-Abad ${ }^{2}$, \\ Kristian Alonso-Stenberg ${ }^{1}(\mathbb{D})$ and Hugh Gash $^{3}(\mathbb{D}$ \\ 1 Department of Applied Mathematics, University of Alicante, 03690 Alicante, Spain; \\ joseluisusodomenech@gmail.com (J.-L.U.-D.); kristian.alonso@ua.es (K.A.-S.) \\ 2 Department of Mathematics, University of Alicante, 03690 Alicante, Spain; lorena.segura@ua.es \\ 3 Institute of Education, Dublin City University, D09 Y18 Dublin, Ireland; hugh.gash@spd.dcu.ie \\ * Correspondence: josue.selva@ua.es; Tel.: +34-680-418-381; Fax: +34-965-909-707
}

Received: 6 February 2020; Accepted: 18 March 2020; Published: 1 April 2020

\begin{abstract}
Cantor thought of the principles of set theory or intuitive principles as universal forms that can apply to any actual or possible totality. This is something, however, which need not be accepted if there are totalities which have a fundamental ontological value and do not conform to these principles. The difficulties involved are not related to ontological problems but with certain peculiar sets, including the set of all sets that are not members of themselves, the set of all sets, and the ordinal of all ordinals. These problematic totalities for intuitive theory can be treated satisfactorily with the Zermelo and Fraenkel (ZF) axioms or the von Neumann, Bernays, and Gödel (NBG) axioms, and the iterative conceptions expressed in them.
\end{abstract}

Keywords: cantorian paradoxes; classes; inconsistent totalities; sets; solutions of extension; solutions of limitation

\section{Introduction}

The Cantorian paradoxes proposed here, are [1]:

C1 There is not a set of all truths.

C2 There is not a set of all states of affairs or facts.

C3 There is not a set of propositions or maximally consistent sentences.

C4 There is not a set of all possible states of affairs.

C5 There is not a set of all essences.

C6 There is no set of all entities.

C7 There is no set of all universals.

What generates Cantorian paradoxes? First, a certain totality (all truths, all the states of things, all essences, all propositions of a maximally consistent set all possible states of affairs, etc.) is selected. Secondly, it happens that the totality selected is "productive" in the sense that given any set of members, new members can always be added. Cantorian paradoxes arise because it is assumed that a given set has a given "fixity or identity" in its members (either finite or infinite, and if it is infinite, is countable or uncountable). Since a set is "fixed", the power set is determined, and must have a cardinal or greater number. The problem here is that the totality selected to be "productive" does not have the "fixity" which a set requires to enter into this type of iterative structure. Cantor's theorem allows discrimination between infinite sets, because even infinite "totalities" can be assigned a certain determination in their elements, which in set theory is the determination of the set in question: the 
conditions of identity of a whole are strictly about extension, for two sets with the same elements are the same set. Once a set is given, some specific elements can define a set of all subsets of the first. This means that the "all" concerned is "already given". In totalities that generate Cantorian paradoxes, however, it happens that the totality concerned is never "given" completely. The totality in question "creates" new members itself. This is what can be termed its "productive" character. There follow two assumptions:

TI A given set $A$ is postulated.

TII There is a generating principle, such that each subset of $A$ can be assigned as an element of $A$.

The combination of TI and TII leads to a contradiction with Cantor's results, whereby the cardinality of the power set of $A$ is greater than the cardinality of $A$ whatever the cardinality of $A$. There are two general ways to solve the paradox and they are directly related to the two cases of type TI and TII that are in the structure of all Cantorian aporia. These possible solutions are:

(a) Solutions of "extension" that is the negation of TI stating that there is a certain "totality" $A$, but it is not a set. This is a solution of "extension", because it provides a way to expand the ways elements can be added that liberates them from the restrictions in set theory. The solutions of extension discussed here have been inspired by the fact that in certain axiomatized set theories a place is left expressly for certain totalities that are not sets, and have been called "classes".

(b) Solutions of "limitation" involving the denial of TII that try to neutralize the generating principle that produces the appearance of "new" elements of $A$ for every subset of $A$. The solutions of limitation hold that the world is not a set.

Others have introduced three general strategies to attack Cantorian paradoxes. For example, Divers [2], pp. 249-256, distinguishes solutions of "limitation" of "proper classes" (which are not sets) and "non maximality". Here they called by Divers as solutions of "limitation" and "non-maximality" as general solutions of limitation within the meaning of (b). This is the effect of solutions that seek to prevent the operation of the generator principle.

We will examine separately each of these general solutions and will argue that while the solution (b) is not recommended as a general solution, and if this is accepted the solution is (a).

\section{Solutions of Extension}

Set theory [3-7], which is a body of mathematical theory used as a "foundation" in all areas of mathematics, seems to say that there is no external "world" as we usually understand it. Indeed, the Cantorian paradoxes, show that there is no set of all truths, there is no set of all states of affairs, and there is no set of all objects (and the set of all properties). It follows that it is not surprising that the set of all propositions of a maximally consistent set (describing how the world could be), the set of all possible states of affairs, and the set of all individual essences can also be declared non-existent. What reason have we to accept these Cantorian paradoxes?

As usually happens with authentic paradoxes, these lines of argument give the feeling that far from showing that something is wrong with the idea of the "world", and the various ways of understanding possible worlds, the paradoxes show that something is wrong with the premises that have led to this amazing result. For it is indeed as amazing that there is no world like this or that Cantor's theorem is valid, as it is incredible to think that there is no movement by Zeno's paradoxes.

The challenge that is posed is to identify what is causing this result that appears incoherent, and contrary to notions that are perfectly acceptable to our ordinary understanding. We will always open the possibility of applying a modus tollens, instead of a modus ponens (or vice versa), if set theory and our ordinary notion of the world are irreparably incompatible. This is exactly what will be done in this work.

The solutions of extension discussed here have been inspired by the fact that in certain axiomatized set theories a place is left expressly for certain totalities that are not sets, and have been called "classes". 
As is well known, there are two alternative ways of axiomatizing set theory [8], pp. 312-316 one by Zermelo and Fraenkel (usually known as ZF) and the other by von Neumann, Bernays, and Gödel (NBG). In NBG there is the concept of a "class" which cannot be a set. A class that is not ordinarily a set is called a "proper class". Von Neumann, defines sets as a kind of class and thus leaves the door open to consider classes of objects that are "too big" to be considered a set, for example the class of all objects and all sets. Cantor had spoken instead of consistent pluralities and "inconsistent" pluralities; this is exactly the pluralities that are too large for set operations.

Cantorian paradoxes led to the development of the axiomatization of set theory, and in particular, the conception of a set with an iterative function. In this conception a set functions in accordance with operations that then can be applied iteratively on the sets already defined. A well formulated formula does not define a set; that is, the set of all objects that satisfy the formula, without assuming that these objects are already part of a set. For example, the famous axiom of separation of Zermelo (Axiom III in his axiomatization of 1908) states that a formula $\phi$, is defined as the set of all $x$ objects that satisfy $\phi,\{x: \phi x\}$ iff there is a set $y$ such that $(x \in y \wedge \phi x)$ (see note A1 in Appendix A). Not all well-formed expressions in a language define a set if the set in question is not already given in the hierarchy of sets iteratively generated by the operations specified in the axioms. Thus, it is not clear in the expression the "set of all sets" that there is a set of all objects that satisfy that formula. In fact, this set does not exist.

The way in which von Neumann $[9,10]$ deals with this issue is totally different. Instead of declaring the absence of certain totalities (being inconsistent), he defines sets as entities that meet certain conditions, and that the usual iterative principles are applicable. Von Neumann did not need to exorcise existence totalities as "too big" to specify the iterative set structure. The primitive notion with which "set" is defined in his axiomatization is "function". We distinguish between "things-I" and "things -II" (Dinge-I, Dinge-II):

1. Things-I are the arguments on which the function can be applied, i.e., the "arguments".

2. Things-II are functions. A function can also be an argument of another function and is called a "thing-I/ II" or "function-argument".

The argument is the value of the function for the argument " $a$ ", and is expressed as $[f, a]$. Here a "set" is defined as follows:

Definition 1. A "domain" is a function $f$ such that for every argument $x$, or $[f, x]=0$ or $[f, x]=1$.

Here, 0 and 1 are elements that indicate intuitively that something satisfies the function $f$, or do not satisfy the function.

Definition 2. It is said of a domain $f$ that it is a set if $f$ is a function argument.

The expression " $a \in f$ " is an abbreviation of $[f, a] \neq 0$, where $f$ is a function and " $a$ " is an argument.

Intuitively, a set is selected as:

1. The collection of objects that satisfy a certain function (i.e., the collection of objects such that, for a given function $f,[f, x]=1$ ).

2. It is an argument of another function.

According to these definitions, it is possible that there is some function $f$ such that there is another function $g$ and $f \in g$. Such a function $f$ selects a totality of elements that is not a set (see note A2). Here we are saying that a function $f$ is not a function-argument, that is, it is not an argument to another function (it is not a set) if and only if all elements of $f$ can be applied in the domain of all arguments. It is not necessary that the application of the elements of $f$ in the universal collection of 
all the arguments is such that each different element of $f$ will be assigned a different element of this universal collection (i.e., is not an injective function). However, it is required that each element of the universal collection is assigned to an element of $f$ (that is, is a surjective function). This ensures that the domain defined by the function $f$ must be at least as large as the universal collection of all arguments.

Axiom IV.2 (see note A3) is crucial to the idea of what is meant as "proper class" or "proper domain": According to the definitions given, the axiom IV.2 expresses a certain function $f$ (in von Neumann's formulation and is not a function-argument and therefore is not a set) if and only if it satisfies the following condition:

Condition 1. For each object $x$, whether it is a set or not, there is a function $g$ ( $b$ in von Neumann's formulation) such that each element $y$ of $f$ (that is, each object $y$, such that $[f, y]=1$, or in other words, such that $y \in f$ ) is assigned $x$.

Definition 3. It is said of a domain that it is a proper domain if there is a function that applies to that domain on the universe of all the arguments, composed of objects and sets.

Consequence 1. A domain ceases to be a set when it is "as big" as the collection of all ordinary objects, consisting of sets and elements of the sets (see note A4).

What can we say about these different axiomatizations with respect to the Cantorian paradoxes? In principle, the two axiomatizations are equivalent in the sense that everything that can be tested with one of them can also be tested with the other. There is no reason to prefer one over the other with regard to questions of set theory. This does not preclude consideration from a viewpoint external to the purely set-theoretic to make one more preferable for example for reasons of ontological perspicuity. The obvious solution would argue here that the "world" (and then, by extension, a "possible world") is entirely too big to be a whole and should be taken as a class by itself, in the sense that this expression has in NBG. This assumption, however, has been rejected by some philosophers, like Christopher Menzel [11]. The fundamental reason for denying that the world can be conceived as a class by itself in the sense of NBG, it is that in these set-theoretic concepts, the difference between set and class is given not only by a consideration due to the "size" of the two types but also because of a structural difference.

In the iterative design, sets only exist to be "built" by operations on an initial collection of basic elements (urelements: $\varnothing$ and any other atoms that are given): union, parity, power set, separation and replacement.

Given the initial collection of urelements, and these operations, a cumulative hierarchy of levels, is generated each of which is assigned an ordinal number, and contains all the collections that can be formed at the previous level in this hierarchy. Here a proper class is outside of this cumulative hierarchy precisely because it is the ultimate result of this process of building sets. Thus, a proper class is to be distinguished not only by an exaggerated size, but also by an exorbitantly complex internal structure. A proper class is "beyond" any set operations that could build or generate and, therefore, has a completely different nature to a set.

Consider the set of all true propositions. This set comprises only propositions which are not sets and which have a range of 0 . The set of all true propositions has, then, a perfectly clear range of 1 (the range immediately above the range 0 in the set structure). It proves that the set of all true propositions cannot be said to be a proper class in the sense of NBG. Something similar could be said of the set of all states of affairs, the set of all objects or the set of all properties. Grim [12] makes note also that any proper class, which cannot be included in other "big" classes, is not acceptable for a theorist who wants to talk about the class of all truths. So for example, Grim is interested in stating that the class of all true propositions is part of the class of all propositions.

However, these peculiarities of a proper class in the NBG axiomatizations are not really an obstacle to the development of solutions of extension. The problem that is discussed here is whether the Cantorian results are taken as a substantive ontological limitation in the "size" of the entities that 
can be postulated, that is, if Cantor's result should be taken as a sufficient reason to reject the existence of the world. In particular, in what sense must it be said that a certain totality is "inconsistent" as Cantor has argued?

Definition 4. A totality is "inconsistent" when given this totality and given the usual set operations one can deduce an explicit inconsistency.

In all Cantorian arguments which have been considered it has been possible to determine precisely the same structure. The postulation of a certain totality as a set allows deducing a conclusion in open contradiction to Cantor's theorem. When speaking of an "inconsistent" totality, it is clear that there is a reason to reject the postulation of such a totality. We have already seen how von Neumann has avoided talking about such "inconsistent" totalities by the process of differentiating sets as part of the iterative structure of the "proper classes." The way proper classes are defined in NBG however, is restricted to the kind of paradoxes that have been taken directly into account by the axiomatization of set theory.

It can be stated in a legitimate way, that the ontological problems discussed here, which have been called Cantorian paradoxes are difficulties requiring introducing limitations on the scope of the axioms. It does not matter if these totalities cannot be considered "proper classes" according to what could be expected in NBG. That is, if we maintain that a certain totality is a set and this leads to a contradiction, then it can be rejected as a totality but may also be rejected as a set. The Cantorian paradoxes considered so far can be thought of as depending on a premise of this kind, which is a paraphrase of the thesis TI such as indicated above:

\section{TI' There is a totality $A$. \\ $\mathrm{TI}^{\prime \prime} \quad A$ is a set.}

We reject the thesis TI" under the Cantorian argument, but do not reject the thesis TI' according to which there is a certain totality $A$. Now it might be objected that this assumption is unreasonable, that is, it is motivated simply to avoid uncomfortable ontological conclusions. Indeed, one could say that the principles of set theory, collected in the axioms are plausible independently or, at least, seem to be independently plausible.

It seems natural to think that given a collection of objects, we can define the collection of all collections formed from elements of the original collection. Furthermore, it seems natural to think that, no matter what the size of the collection in question, the new collection defined by the operation of power set will have a greater cardinality. This occurs when the collection is finite and nothing appears to prevent this thesis generalize to infinite collections of objects. Why cannot these principles be applied to totalities like the world? What kind of prohibition may prevent us trying to think of the world with the same principles that we thought about any collection? The answer we are proposing here is that the denial of certain totalities are sets is the most reasonable solution for a theoretically very unsatisfactory situation, even though this is not an alternative contemplated by existing axiomatizations.

Consider again the dialectical situation. The postulation that the world exists as a set of all true propositions, or the set of all states of all objects or things, leads to an explicit contradiction with Cantor's theorem. This, given a generator principle for these totalities which allows the introduction of elements in the set in question for each sub-set of it. It would be unreasonable to declare all set theory false on the basis of this result. An idea of this kind, for example, has drawn attention to theories of non-standard sets in which the axiom of power set or at least not worth with all generality. Of course, from a purely formal point of view, it is interesting to consider what happens when a postulate is eliminated within a set of independent postulates, but there is no reason to believe that all set theory should be considered without the power set axiom. Although these non-standard systems exist, this does not prevent the existence of standard systems in which this axiom is valid. To avoid the paradoxes, one could argue that the theory of sets is only valid when there is no power set but this is 
completely unreasonable. Standard systems are what give Cantor's paradise, otherwise it's unrealistic to think that we mathematicians are going to give them up for a metaphysical pruritus.

It would also be unreasonable to state that there is no world because this mathematical result contradicts Cantor's theorem. What choice do we have? There are solutions of limitation, or the solution as advocated in this paper: simply hold that the world is not a set.

The main independent justification having the general solution proposed is that the axioms of set theory, and the iterative conception expressed by them are not natural. It is not a series of assumptions that is obvious to common sense. The power set axiom can be "natural" in the sense of looking like a perfectly acceptable assumption to any rational person, whether or not mathematically sophisticated. This may also happen with the axiom of extensionality or determination, with the parity axiom or the axiom of union). However, it may not always be so with other languages, nor with concepts such as separation, replacement, the infinite, perhaps also with the axiom of choice, nor with the application of a mysterious mathematical entity: $\varnothing$ whose formal utility is obvious, that the set operations remain always defined, but one cannot claim that it is obvious to common sense (see note A5). The only reason that seems to justify an axiom like the separation axiom (see note A6) is to prevent the emergence of paradoxes such as Russell's paradox [13] (see note A7).

This is not a natural assumption in any way. It is a reasonable way to provide a consistent theory, as far as we know, which includes all central insights of Cantor and allows one to inhabit his paradise with reasonable confidence, but it is not a set of principles, without contradiction, for all thinkable collections. The set-theoretic structures are designed for specific theoretical purposes. In such structures, infinite totalities with different cardinalities are made thinkable, and thinkable infinitudes become arithmetic and are different and commensurate with each other. However, clearly this is not about the principles that make thinkable all collection of objects in general. In fact, we do not think about collections of objects usually as part of the set-theoretic structure. Well, if so, is it so difficult to accept that the world is not a set, that is, an object of this theory? What we are claiming when we say that the world is not simply a set, is that the entire apparatus of set theory should not apply. A "set" is a type of entity that meets very specific requirements and we will think about the world as an entity of that characteristic species. Nor is the world a class, in the sense of NBG, but it can be argued that the denial of the world to be a "set" is a maneuver inspired by von Neumann's spirit, although this idea in particular cannot be attributed to him.

The crucial point is that it is not reasonable to argue that the axioms of set theory are the measure of what is in the Universe. It is not reasonable to argue that an entity can only exist if it has a place in the hierarchy of sets, that is, if it has been generated by set operations. It is not credible to argue that the power set axiom will be the keystone of our fundamental ontology, functioning as the discriminator of what exists. The postulation of the world, as the totality of all entities, or totality of all states of affairs (and the correlative postulation of the totality of all true propositions), has sufficient independent guarantees, so that we reject any argument against it, as a reduction of the assumptions leading to that result.

It is instructive in this regard, to compare these considerations with those defended by T. Williamson [14]. Williamson advocated the need for unrestricted quantification over "all" as a requirement of our rationality, despite the logical Cantorian pruritus. This implies that set theory is a form of mathematical structure being formally very simple and powerful (and therefore interesting for mathematicians), but not our fundamental ontology. Of course, this seems to go against the express wishes of Cantor, but Cantor's original idea faded anyway. Cantor left unresolved two issues concerning the sufficiency of transfinite arithmetic to "measure all of the crowds of the universe": the problem of the continuum, and the problem of good order. The first Cohen proved to be un-demonstrable from the remaining usual set-theoretic axioms in 1963. The second has been resolved by introducing the axiom of choice and has been less intuitive to some than the remaining postulates of set theory. 


\section{Solutions of Limitation}

We will see that the solutions of limitation imposed such restrictions that it is not credible to believe that they can provide answers to all Cantorian paradoxes and, especially, to Cantorian paradoxes with ontological implications. As we have seen, Cantorian paradoxes arise from two assumptions. On the one hand, it is assumed that there is some determined totality $A$ (TI), and then there is a generator principle, which increases the elements of $A$ to make it as "big" as the power set of $A$ (TII).

The extension solutions seek to deny the assumption TI, rejecting that the totality in question be considered a set, without having to deny the existence of the totality $A$. These limitations of these solutions however, seek to restrict the operation of the generator principle which increases the elements of $A$. Consider, for example, what happens in the Cantorian paradox C1. Consider the existence of the set $A$ of all truths. For each subset of $A$, there is a new true proposition that should also be a truth and, therefore, should be part of $A$. $A$ is as big as its power set. The generator principle here works formulating true propositions for each sub-set of the set $A$. The Cantorian paradox case $\mathrm{C} 2$ is very similar to this, only true propositions must be replaced here by states of affairs or facts. For the Cantorian paradox C6 on the set of all entities, the generating principle is explicitly specified as the mereological principle that, given two entities $x$ and $y$, there is the mereological sum $(x+y)$. Limitation solutions are ways to prevent those new entities being added to the set in question for each of its sub-sets. For this it is essential that the totality that has been defined is restricted in a precise sense. Limitation solutions seek to effect some "stratification" of the elements, which will make up the totality in question, so that the new elements that can be generated for each subset are excluded by corresponding to a higher "level" (see note A8). Consider the question, firstly, with respect to the set of all true propositions. In the first place, a set of all objects $\left\{x_{1}, x_{2}, \ldots, x_{n}\right\}$ is defined (see note A9) and also a set of all the properties of the first order $\left\{P_{1}, P_{2}, \ldots, P_{n}\right\}$ (see notes A10 and A11). Given these initial sets of objects and properties, we can define a set of all propositions at level-0, which only deal with properties and relationships between objects, $\left\{P_{1} x_{1}, P_{1} x_{2}, \ldots, P_{1} x_{n}, P_{2} x_{1}, P_{2} x_{2}, \ldots, P_{2} x_{n}, \ldots, P_{n} x_{1}, P_{n} x_{2}, \ldots, P_{n} x_{n}\right\}$. A subset of these propositions at level- 0 is the set of all true propositions. Let $A$ be the set of all true propositions. It is obvious that every subset of $A$ can be considered as a true proposition. The idea is to define the level of such propositions that fall outside the set $A$. Level-1 will now be defined as the level of all propositions that deal with propositions at level-0. Given the way the argument $A$ to a given subset of $A$ was formulated, $\left\{p_{1}, p_{2}\right\}$ may be considered a true proposition, as $p_{1} \in\left[p_{1}, p_{2}\right]$. If $p_{1}$ and $p_{2}$ are propositions at level- 0 , that is, they are propositions that are enunciating that certain objects have certain properties of the first order, the proposition stating that $p_{1}$ belongs to the set consisting of the propositions $p_{1}$ and $p_{2}$ is a level-1 proposition that deals with level-0 propositions (see note A12). Then, they can be defined as propositions at a higher level.

Level-2 propositions are propositions that deal with level-1 propositions. Level-3 propositions deal level-2 propositions, and so on. For each of these levels, moreover, it is necessary to define a particular set of properties at different levels.

The set of first-order properties used to define level-0 propositions is composed only of properties that are predicated of objects, i.e., properties of the first level. The properties used to define level-1 are a set of properties that are predicated of propositions at level- 0 , for example, the property of being an element of the set $\left\{p_{1}, p_{2}\right\}$, which is a property of the second level. Then there will be third-level properties that are predicated of propositions at level-2 (which in turn, specify properties of propositions at the second level of level-1), etc.

Thus the Cantorian paradox C1 is resolved. The problem, however, is that this solution is useless if the remaining Cantorian paradoxes C2, C6 and C7 are not also resolved. By solving the initial problem of the set of all true propositions, this solution has not yet saved the "world", because this solution should appeal to the set of all objects that are affected by the paradox $\mathrm{C} 6$, and must appeal to the set of all the properties affected by paradox C7. In addition, there remains the problem regarding the set of all states of affairs, that is, paradox C2 (see note A13). 
It happens, then, that if we want to be rid of paradoxes applying to the concept of "the world", it does not help to implement a local solution of stratification. It will not be useful to stratify the states of things, without also having to stratify the propositions, objects and properties. We cannot stratify objects, in short, without having to stratify the properties, states of affairs and propositions. Limitation solutions of stratification can only be global. Otherwise, the Cantorian paradoxes, banished from one area, reappear in another area.

In the case of objects, we must assume that there is a set of atoms at level-0. The mereological sums of atoms will have level-1. The mereological sums of entities from level-1 will be level-2 and so on. Something similar has to apply to the properties. There will be a level of simple properties at level-0. The structural properties formed by character of properties at level-0 are the properties at level-1. Then, in general, the properties formed by properties at level- $[n-1]$ shall be level- $n$.

When it comes to a state of affairs, on the other hand, it will have to define a level- 0 of a basic or atomic state of affairs having to be made up of objects at level-0 and properties at level-0. The upper strata consist of states of affairs of lower levels. All this must then appear in the stratification of the propositions. The result is a general diagram of immense complexity.

If the world is the set of all states of affairs, and is designed to prevent Cantorian paradoxes by means of the solution of limitation, then the world will be limited to the described facts at level- 0 , but the result is then that in the world causal relationships will not appear. This, of course, does not matter to some philosophers whose conception of causality is anti-realistic [15-18], but many other philosophers argue that causal facts about the world are ontologically basic facts and for them this is deeply unsatisfactory [19-22]. However, it could be argued that this is not a major difficulty. Arguably, in fact, the level of states of affairs that must be mentioned to define the world as a set of all the facts must be high enough to include the causal relationships, which is not an arbitrarily high level. This is not so simple, however. This is perhaps what is most doubtful about the limitation solution: if it is to make sense it should apply globally to propositions, states of affairs, objects and properties (and other ontological categories if they are given). However, for this to be so, it must have felt the existence of a level of precise mereological composition for each entity that populates the world and with whom we have regular contact. To assign such a defined level it is necessary to identify a basic level at which only "atoms" are given without parts. Likewise, it is necessary to apply a basic level of unstructured properties.

However, it is obvious that this is a dubious ontological thesis at best. It is unwise to think that to solve the problem of Cantorian paradoxes we need to come out the world to look at real "atoms". If we were to follow this solution, it must be assumed that, given the Cantorian paradoxes, and then there must be a level of atoms in the world, because otherwise we cannot make intelligible the idea of world. This is what seems unreasonable. This does not mean that in the future a definitive and independent philosophical argument will not emerge for the nomination of atoms in the world, or, what may seem more difficult, an atom (i.e., a material object without parts) will be discovered by empirical research. It does not seem an appropriate strategy to rest the defense of the intelligibility of the notion of "world" in the eventual success of such speculation.

\section{Conclusions}

In the present work, we have seen that: (a) extension solutions are based on the fact that certain axiomatized set theories contain a place for totalities that are not sets (called classes); and (b) limitation solutions determine that the world is not a set. When it comes to address the general issue of resolving the problems created by Cantor's paradoxes from the point of view of modal metaphysics, the expansion solution seems the most advisable. This solution postulates simply disregarding Cantor's paradoxes. Set theory should be taken as an interesting mathematical theory, but not as the ultimate ontological model of what exists in the universe. Surely it is necessary to have more clarity on the true scope of set-theoretic structures, and also, more clarity on what is generally a mathematical entity, to say with certainty that Cantor's results have limited value. It takes much to get this clarity, of course, 
but it does not help to apply the expansion solution as the most plausible, given the knowledge we have today about these issues.

Author Contributions: Conceptualization, J.-A.N.-S., J.-L.U.-D. and L.S.-A.; methodology, J.-A.N.-S., J.-L.U.-D. and L.S.-A.; formal analysis, J.-A.N.-S., J.-L.U.-D. and L.S.-A.; investigation, J.-A.N.-S., J.-L.U.-D. and L.S.-A.; writing - original draft preparation, J.-A.N.-S., J.-L.U.-D., L.S.-A., K.A.-S. and H.G.; writing-review and editing, J.-A.N.-S., K.A.-S. and H.G.; visualization, J.-A.N.-S., J.-L.U.-D., L.S.-A., K.A.-S. and H.G.; supervision, J.-A.N.-S. and J.-L.U.-D.; project administration, J.-A.N.-S. and J.-L.U.-D. All authors have read and agreed to the published version of the manuscript.

Funding: This research received no external funding.

Conflicts of Interest: The authors declare no conflict of interest.

\section{Appendix A. Notes}

A1 Zermelo's axioms (1908) are: (I) Determination Axiom: two sets are identical if and only if they have the same elements; (II) Axiom of elementary sets: there is an empty set $\varnothing$, which has no elements; (III) Axiom of separation: as noted above; (IV) Power Set Axiom: given a set $x$, there exists a set $P(x)$, which has as elements all subsets of $x ;(\mathrm{V})$ Axiom of union: if there is a set $x$, then there is a set $U x$ union, composed of all the elements of the elements of $x$; (VI) Selection Axiom: if $x$ is a set having as elements, non-empty disjoint sets (that is, without common elements), then the set $U x$ union includes a set in which elements are contained as one and only one element each of the sub-sets of non-empty disjoint of $x$; (VII) Axiom of infinity: there is a set $X$ such that: (1) $\varnothing \in X$, and (2) if $x \in X$, then $\{x\} \in X$ (which generates an infinite sequence $\varnothing,\{\varnothing\},\{\{\varnothing\}\}$, etc.).

A2 Recall that a "function" or "application" is defined as a relationship between two collections of objects $A$ and $B$, such that elements of $A$ (usually called the domain of the function), which can be one or more, assigned one and only one element of $B$ (usually called travel or reverse domain of the function).

A3 Axiom IV.2: A thing-II $a$ it is not a thing I/II iff there is a thing-II $b$ such that for every thing-I $x$ there is a $y$ that meets the conditions $[a, y] \neq A$ and $[b, y]=x$.

A4 To say here that "not a set" is saying it is not an argument to another function $y$, therefore, it is not an element of another set. In other words, a "too big" domain is automatically out of the iterative structure. There is here no need to declare these "inconsistent" domains to develop a restricted to a certain class of mathematical objects, namely, the sets, the theory that defines the usual properties.

A5 The empty or null set is a strange concept. It is a set, but it has no members. This is of course not a contingent fact about it, but one bound up with its very identity: the null set is essentially null. Intuitively, however, one might have thought that a set is a group of two or more things. Indeed, Georg Cantor famously defines a set (Menge) as "any collection into a whole (Zusammenfassung zu einem Ganzen) of definite and separate objects of our intuition and thought" [23], p. 85. In the case of the null set, however, there are no definite objects that it collects. So in what sense is the null set a set? Set theory can be done either naively or axiomatically. In the standard axiomatic approach to the subject, that of Zermelo-Fraenkel, the existence of the null set is posited in a special axiom. In Zermelo's 1908 formulation, both the null set and singleton sets are posited in his Axiom der Elementarmengen. About the null set Zermelo writes, "There exists a (fictitious) set, the null set, 0 that contains no element at all" [24], p. 202. One curious feature of this Zermelian formulation is that "fictitious" appears to cancel out exists. To exist, if it means anything, is to exist in reality, in splendid independence of language and mind. Something that exists as a fiction precisely does not exist. There is a more serious problem. Intuitively, the existence of a set depends on the existence of its members. It exists because the elements exist, not vice versa. In the case of the null set, however, there is nothing on which the null set can depend for its existence. Bertrand Russell refers to the difficulty in his early Principles of Mathematics. In Whitehead and Russell's Principia Mathematica, we learn that "to say that a class exists is equivalent to saying that the class 
is not equal to the null-class" [13]. It seems to follow from this that the null set does not exist. However, two sets $A$ and $B$ are said to be disjoint if they have no members in common. What then is the intersection of two disjoint sets? The intersection of any two sets is a set. We also see the intersection of $A^{\prime}$ and $B^{\prime}$ (also disjoint) to be the same as the intersection of $A$ and $B$. Thus we speak of the null set, where "the" connotes uniqueness. The Union Axiom states that, given any set $A$, there exists a set $U A$ the members of which are exactly the members of the members of $A$. Now suppose we apply the Union Axiom to the set $\left\{x_{1}, x_{2}\right\}$. Since the members of this set do not have members, $U\left\{x_{1}, x_{2}\right\}=$ the null set. In general, the application of the Union Axiom to any set the members of which are nonsets yields the null set. The uniqueness of the null set can be proven by reductio ad absurdum. In such a mode of proof one attempt to show that a certain assumption, in the presence of propositions antecedently accepted, implies a contradiction. So assume that the null set is not unique: assume that there are two null sets, $A$ and $A^{\prime}$. Then, by the Axiom of Extensionality (two sets are the same iff they have all the same members), $N$ has a member that $A^{\prime}$ does not have, or vice versa. However, this issue is in a contradiction inasmuch as neither $A$ nor $A^{\prime}$ has a member. Therefore, the null set is unique. Quine notices that in New Foundations one can prove the existence of the empty set, the set containing the empty set, the set containing the set containing the empty set, and so on [25].

A6 The axiom of separation can introduce the set of all $x$ objects that satisfy a condition $\phi\{x ; \phi x\}$ iff there is a set $y$, such that $(x \in y \wedge \phi x)$. That is, a set only exists if there is already another set. Sets only exist within the hierarchy of sets, as elements of other sets.

A7 Russell's paradox, which he published in Principles of Mathematics in 1903, demonstrated a fundamental limitation of such a system [13]. In modern terms, this sort of system is best described in terms of sets, using so-called set-builder notation. For example, we can describe the collection of numbers 4,5 and 6 by saying that $x$ is the collection of integers, represented by $n$, that are greater than 3 and less than 7 . We write this description of the set formally as $x=\{n: n$ is an integer and $3<n<7\}$. The objects in the set don't have to be numbers. We might let $y=\{x: x$ is a male resident of the United States $\}$. Seemingly, any description of $x$ could fill the space after the colon. However, Russell (and independently, Ernst Zermelo) noticed that $x=\{a: a$ is not in $a\}$ leads to a contradiction in the same way as the description of the collection of barbers. Is $x$ itself in the set $x$ ? Either answer leads to a contradiction.

A8 The traditional theory of types of Russell is a way of effecting this stratification.

A9 This assumption is already affected by the Cantorian paradox C6.

A10 This assumption is already affected by the Cantorian paradox $\mathrm{C}$.

A11 It is understood as "first order properties" the properties, possibly $n$-adic, attributed to objects.

A12 For this, is needless to say that the set whose elements are stratum- 0 must be of a higher stratum. Stratification concerns only the propositions and not to entities dealing with these propositions; here a set is simply an entity.

A13 The totality of all the states of things must exist, if is that they must be truthmakers for all true propositions.

\section{References}

1. Usó-Doménech, J.L.; Nescolarde-Selva, J.A.; Segura-Abad, L.; Gash, H.; Alonso-Stenberg, K. Cantor Paradoxes, Possible Worlds and Set Theory. Mathematics 2019, 7, 628. [CrossRef]

2. $\quad$ Divers, J. Possible Worlds, 1st ed.; Routledge: London, UK, 2002; pp. 249-256.

3. Drake, F. Set Theory: An Introduction to Large Cardinals; North-Holland Publishing Company: Amsterdam, The Netherlands, 1974.

4. Fraenkel, A. Abstract Set Theory; North-Holland Publishing Company: Amsterdam, The Netherlands, 1953.

5. Fraenkel, A.; Van Dalen, D.; Levy, A.; Bar-Hillel, Y. Foundations of Set Theory; North-Holland Publishing Company: Amsterdam, The Netherlands, 1973.

6. Levy, A. Basic Set Theory; Springer: Berlin, Germany, 1979. 
7. Quine, W.; Pierce, E. Set Theory and its Logic; Belknap Press of Harvard University Press: Cambridge, MA, USA, 1980.

8. Potter, M. Set Theory and its Philosophy: A Critical Introduction, 1st ed.; Oxford University Press: Oxford, UK, 2004; pp. 312-316.

9. Mendelson, E. Introduction to Mathematical Logic, 4th ed.; Chapman and Hall/CRC: London, UK, 1997.

10. Montague, R. Semantic Closure and Non-Finite Axiomatizability I. In Infinitistic Methods, Proceedings of the Symposium on Foundations of Mathematics, Warsaw, Poland, 2-9 September 1959; Pergamon Press: London, UK, 1961; pp. 45-69.

11. Menzel, C. On Set Theoretic Possible Worlds. Analysis 1986, 46, 68-72. [CrossRef]

12. Grim, P. On Sets and Worlds: A Reply to Menzel. Analysis 1986, 46, 186-191. [CrossRef]

13. Whitehead, A.; Russell, B. Principia Mathematica; Cambridge University Press: Cambridge, UK, 1997.

14. Williamson, T. Everything. Philosophi. Perspect. 2003, 17, 415-465. [CrossRef]

15. Reid, T. Essays on the Active Powers of Man; Garland: New York, NY, USA, 1788.

16. Austin, J. Philosophical Papers; Oxford University Press: Oxford, UK, 1961.

17. Spinoza, B. The Collected Works of Spinoza; Princeton University Press: Princeton, NJ, USA, 1985.

18. Tegmark, M. Our Mathematical Universe: My Quest for the Ultimate Nature of Reality; Knopf: New York, NY, USA, 2014.

19. Esfeld, M. Causal Realism. Probab. Laws Struct. 2012, 157-168._11. [CrossRef]

20. Bird, A. Nature's Metaphysics. Laws and Properties; Oxford University Press: Oxford, UK, 2007.

21. Shoemaker, S. Causality And Properties. Time Cause 1980, 109-135._7. [CrossRef]

22. Hawthorne, J. Causal Structuralism. Metaphysics 2001, 15, 361-378.

23. Cantor, G. Contributions to the Founding of the Theory of Transinfinite Numbers; Dover Publications: New York, NY, USA, 1955; p. 85.

24. Van Heijenoort, J. From Frege to Gödel; Harvard University Press: Cambridge, MA, USA, 1967; p. 202.

25. Specker, E. The Axiom of Choice in Quine's New Foundations for Mathematical Logic. Proc. Natl. Acad. Sci. USA 1953, 39, 972-975. [CrossRef] [PubMed]

(C) 2020 by the authors. Licensee MDPI, Basel, Switzerland. This article is an open access article distributed under the terms and conditions of the Creative Commons Attribution (CC BY) license (http:/ / creativecommons.org/licenses/by/4.0/). 